\title{
DOCTRINA
}

\section{La participación ciudadana en los planes de descontaminación atmosférica. Una evaluación sin romance}

\author{
Citizen participation in air pollution plans. An assessment without romance
}

\author{
Luis Cordero Vega (iD y Ximena Insunza Corvalán (iD \\ Universidad de Chile
}

RESUMEN Este es un trabajo exploratorio que tiene por finalidad analizar la participación ciudadana como mecanismo de incidencia en la definición de actos administrativos o políticas públicas. Para ello, se estudia el proceso de elaboración de los planes de prevención y/o descontaminación como instrumentos que manifiestan un problema global en un territorio donde resulta indispensable la adopción de medidas y en que la incidencia de la participación debería ser gravitante y efectiva. Con tal finalidad, se expone el marco regulatorio nacional, el proceso de elaboración de los planes y los resultados de la participación, demostrando que no existe consistencia entre lo normativamente pretendido y la implementación de los procedimientos participativos.

PALABRAS CLAVE Planes de descontaminación, contaminación atmosférica, participación ciudadana, procedimiento administrativo.

ABSTRACT This is an exploratory work that aims to analyze citizen participation as a mechanism to influence the definition of administrative acts or public policies. To this end, the process of development of Prevention and Decontamination Plans is examined, as instruments that manifest a global problem in a territory where the adoption of effective measures is essential and where the impact of participation should be conceptually influential and effective. As a result of the research, it is like to affirm that, the national regulatory framework, the process of drawing up the Plans and the results of participation are set out, demonstrating that there is no consistency between what is normatively intended with the implementation of participatory procedures.

KEYWORDS Decontamination plans, air pollution, citizen participation, administrative process. 


\section{Antecedentes}

Los planes de prevención y/o descontaminación son instrumentos de gestión ambiental complejos, en virtud de los cuales se adoptan un conjunto de medidas administrativas específicas con el propósito de que una determinada zona del territorio retorne a los estándares ambientales establecidos previamente mediante una norma de calidad ambiental. ${ }^{1}$ Esto explica que existan dos tipos de planes: de prevención, cuando la norma está en zona de latencia, y de descontaminación, cuando la norma de calidad ha sido superada y así se acredita. Ambos pueden concurrir en una misma regulación (Bermúdez Soto, 2015: 241; Astorga Jorquera, 2014: 339; Guzmán Rosen, 2012: 169).

$\mathrm{Su}$ origen normativo es la Ley 19.300 sobre Bases Generales del Medio Ambiente de 1994, y aunque es un instrumento que se puede utilizar para múltiples componentes ambientales, desde la dictación de esta ley se ha concentrado específicamente en contaminación atmosférica. En la actualidad disponemos de 25 planes vigentes ${ }^{2}$ que buscan recuperar los estándares de calidad ambiental.

La regulación de los planes de prevención y/o descontaminación heredó la gestión ambiental utilizada a través del Código Sanitario que, bajo la regla de normas de amplia cobertura para proteger la salud humana, dispuso de criterios que se utilizaron como estándares de calidad ${ }^{3}$ y sobre esa base se dictaron los primeros planes de descontaminación focalizados en megafuentes, especialmente en la industria minera. ${ }^{4}$ Las regulaciones ambientales que se dictaron a partir de 1996 reconocieron esas normas y planes, dotándolos en varios casos de continuidad (OCDE y Cepal, 2005: 39; Corporación Tiempo 2000, 1999).

1. La regulación de los planes se encuentra en los artículos 43 a 47 de la Ley 19.300, de 1994, y el Decreto Supremo 39, de 2012, del Ministerio del Medio Ambiente, que aprobó el reglamento para la dictación de los Planes de Prevención y Descontaminación.

2. Por vigencia entendemos aquellos exigibles y sujetos a fiscalización. Véase http://snifa.sma.gob.cl/ v2/Instrumento/Tipo/4 .

3. La regla de competencia general del Código Sanitario, vigente hasta hoy, es el artículo 89. Las normas originales sobre contaminantes atmosféricos se establecieron en el Decreto Supremo 144, de 1961, del Ministerio de Salud. A su vez, la Resolución 1215, de 1978, del Ministerio de Salud, permitió regular por primera vez las normas de calidad y de emisión. Sus reglas permitieron la dictación de los primeros planes de descontaminación.

4. Son los casos, por ejemplo, de los planes de Huasco, Fundición Hernán Videla Lira de Enami, Fundición de Potrerillos de la División El Salvador de Codelco y Fundición Chuquicamata. La regulación sistemática de esto se realizó en 1991 mediante el Decreto Supremo 185 del Ministerio de Minería, que reglamentó el funcionamiento de establecimientos emisores de anhídrido sulfuroso, material particulado y arsénico en todo el territorio nacional. Esta norma originalmente tenía mayores pretensiones dado que reguló en su Título 2 las normas de calidad primaria de material particulado y $\mathrm{SO}_{2}$, derogando la Resolución 1215, y además en su Título 3 estableció zonas de monitoreo de calidad de aire. 
Una de las pretensiones de la regulación ambiental implementada en 1994 es que sus instrumentos, incluidos los planes de prevención y/o descontaminación, descansaran en el principio participativo, uno de sus elementos vitales según sostenía el mensaje del proyecto de ley. ${ }^{5}$ Ese criterio ha sido el recogido por la Corte Suprema en múltiples oportunidades como un principio guía de la gestión de la autoridad ambiental para sus procedimientos administrativos; ${ }^{6} \mathrm{y}$ de igual forma ha sido asumido por la doctrina con total naturalidad como un elemento que permite lograr la «mayor legitimidad social de los planes aprobados» (Guzmán Rosen, 2012: 176) y, a su vez, ha sido reconocido como un elemento propio de la justicia ambiental (Hervé Espejo, 2010: 21; Soto Barrientos y Costa Cordella, 2019: 246), al margen del genuino impacto para cumplir con la pretensión normativa perseguida por ella, es decir, que en los hechos garantice estándares de información y legitimidad socialmente aceptables.

Esa pretensión, mayoritariamente común a la idea de regulación ambiental, no es coherente con la forma y modo en que opera su implementación administrativa. Eso tiene consecuencias significativas porque no solo afecta la legitimidad de las decisiones y su efectividad, sino que lleva a que el derecho descanse simplemente en la utilidad de su declaración, eludiendo las consecuencias de su ejecución.

Para poder fundar esta afirmación, analizaremos a continuación los presupuestos centrales sobre los cuales recae tradicionalmente la idea de participación ciudadana en materia ambiental; luego ilustraremos las etapas participativas en los planes de prevención y/o descontaminación, caracterizando quienes tienen derecho a participar y quienes efectivamente participan; finalmente, expresaremos nuestras conclusiones sobre lo que implica la disfunción entre pretensión y aplicación normativa. Con tal finalidad, utilizaremos los datos de la última generación de planes de prevención y/o descontaminación. ${ }^{7}$

5. El Mensaje propone el principio de participación sobre la base del derecho a informarse, a impugnar proyectos ambientales que afectaban a las comunidades, composición del consejo consultivo, decisión descentralizada de los proyectos en el sistema de evaluación de impacto ambiental, educación y consulta pública en la elaboración de las normas ambientales. Véase la Historia de la Ley 19.300, disponible en https://bit.ly/3dfnHki.

6. Véase, por ejemplo, Soto con Servicio de Evaluación Ambiental (2019), Corte Suprema, 15 de mayo de 2019, rol 197-2019 (considerando 17); Comité Prodefensa del Patrimonio Histórico Cultural de Viña del Mar con Comité de Ministros (2017), Corte Suprema, 6 de noviembre de 2017, rol 97.792-2016 (considerandos 34 y 35); Corporación Fiscalía del Medio Ambiente con Director Ejecutivo del Servicio de Evaluación Ambiental (2017), Corte Suprema, 25 de julio de 2017, rol 31.176-2016 (considerando séptimo); Stipicic con Director Ejecutivo del Servicio de Evaluación Ambiental (2017), Corte Suprema, 16 de marzo de 2017, rol 55.203-2016 (considerandos séptimo y octavo).

7. Metodológicamente hemos organizado la estructura de los planes en tres macrozonas: norte, central y sur. La primera comprende a los planes de Tocopilla, Andacollo y Huasco. La macrozona central corresponde a los planes de Santiago y Quintero-Puchuncaví-Ventanas. Por último, la macrozona sur 


\section{La participación ciudadana}

Existe una abundante literatura que señala que la participación en gestión pública es consecuencia de un paradigma de redistribución del poder entre el Estado y los ciudadanos, de modo que el establecimiento de mecanismos de estas características permite desarrollar verdaderos «consejos de gestores públicos» (Pintaudi, 2005). Este asunto ha sido especialmente relevante en el establecimiento de estructuras estables en la Administración Pública (por ejemplo, en los consejos consultivos, consejos directivos integrados por representantes de la comunidad) o bien en la generación de mecanismos de participación para la toma de la decisión administrativa (regulación urbana, audiencias públicas previas a decisiones generales, pavimentación, elaboración de presupuestos, entre otras).

La discusión evidente que genera este fenómeno es la tensión entre democracia representativa y responsabilidad pública. El resultado ha terminado siendo el establecimiento de mecanismos de participación que se focalizan en instrumentos de gestión, habitualmente de planificación general, como son los planes de prevención y/o descontaminación. ¿Cuál es el mejor equilibrio entre responsabilidad y democracia? Es una de las interrogantes que aún se debate en la literatura de participación.

El motivo de ese debate es consecuencia, además, de dos factores: por un lado, que las decisiones sustantivas de interés general han quedado en mano de organismos administrativos y no de los parlamentos, y enseguida, que las sociedades necesitan tomar en cada caso una decisión colectiva, que esté investida de autoridad y legitimidad (Linares, 2017: 34). Las sociedades deben adoptar decisiones, porque siempre existirá una decisión (principio de inevitabilidad) cuando se trate de asuntos públicos.

Por su parte, la participación ciudadana en materia ambiental es una modalidad de participación en gestión pública que está determinada por la discusión sobre democracia y justicia ambiental como elementos distintivos (Walker, 2012: 42). La mayoría de las decisiones ambientales conllevan la afectación de bienes colectivos bajo lógicas muy distintas a la participación ciudadana en gestión pública, porque las dificultades de participación evidencian serios problemas de inequidad ambiental, por lo que su omisión o sistemas de participación mal diseñados pueden terminar profundizando conflictos distributivos, que resultan indispensables identificar para abordar la conflictividad que afecta a la gobernabilidad actual y futura de las comunidades y territorios (Durán y otros, 2017; Innes y Booher 2004).

La literatura señala como métodos de participación: i) los plebiscitos, ii) las audiencias o consultas públicas sin obligación de respuesta, iii) las consultas públicas

incorpora a los planes de Rancagua, Talca-Maule, Curicó, Concepción, Chillán, Los Ángeles, Temuco, Valdivia, Osorno y Coyhaique. 
en las que es imperativa la respuesta y su consideración, iv) la existencia de comités asesores o consultivos, v) los procesos normativos negociados o la configuración de paneles o jurados ciudadanos, entre otros (Rowe y Frewer, 2000: 8).

Por su parte, existen pocos estudios empíricos que expliquen los resultados de la participación pública en términos de si mejora o no los procesos de formulación de políticas públicas. Sin embargo, sí se han concentrado en identificar cualitativamente cinco elementos que permiten explicar el rol transformador de la participación ciudadana en cuanto proceso. Estos son: a) liderazgo en el proceso participativo; b) un enfoque holístico de la política pública sometida a participación; c) amplia visibilidad al proceso participativo; d) la capacidad de impacto de los ciudadanos en las políticas públicas a través del proceso de participación; y e) el cambio cultural en políticos, técnicos y ciudadanos (Parés y otros, 2014: 115).

De acuerdo con lo anterior, cabe destacar que en nuestra legislación ambiental, mientras el procedimiento de participación ciudadana en el Sistema de Evaluación de Impacto Ambiental (SEIA) exige respuesta fundada de la autoridad que luego le otorga legitimación para reclamar administrativa y judicialmente, ${ }^{8}$ en el caso de los planes de prevención y/o descontaminación, al igual que en el proceso de elaboración de normas, la consulta pública no tiene asociado un deber de respuesta en el procedimiento, ${ }^{9}$ sin perjuicio de que puede impugnar el acto administrativo cualquier persona que estime que no se cumple con lo dispuesto en la Ley 19.300 sobre Bases Generales del Medio Ambiente y le cause perjuicio, ${ }^{10}$ para lo cual es irrelevante la participación en el procedimiento de elaboración del plan.

La falta de consideración de las observaciones, en nuestra opinión, tiene consecuencias en la implementación de los procesos participativos y en la efectividad de las medidas. Se estima que un sistema de participación amplio genera beneficios en la medida que mejora la calidad de las decisiones, contribuye a resolver problemas ambientales concretos, promueve la ciudadanía ambiental, mejora la legitimidad del proceso de decisión y permite la elección de valores públicos que en casos concretos se desea promover, mejorando con todo ello el cumplimiento de las regulaciones (Lee y Abbot, 2003: 80). Muchos de estos supuestos no están presentes en los planes.

El asunto es aún más complejo si se considera que, si bien la regulación permite que personas naturales o jurídicas puedan formular observaciones durante la etapa de consulta pública del anteproyecto de plan, tienen la obligación de acompañar «antecedentes en los que se sustentan» sus observaciones y estos deben ser de «naturaleza técnica, científica, social, económica y jurídica». ${ }^{11}$ El presupuesto de esta

8. Artículo 17 número 6 de la Ley 20.600.

9. Artículos 12 y 13 del Decreto Supremo 39, de 2012.

10. Artículo 18 número 1 de la Ley 20.600.

11. Artículo 12 del mencionado Decreto Supremo 39. 
disposición opera jurídicamente como una barrera para la participación tal como lo analizaremos más adelante, aun cuando la autoridad prescinda, en los hechos, de esa exigencia normativa.

\section{Las estaciones «participativas» de los planes}

Para efectos de evaluar cómo opera la participación en los planes de prevención y/o descontaminación, hemos distinguido entre estaciones formales e informales. Las primeras son aquellas en que el sistema legal considera expresamente algún mecanismo participativo, por ejemplo, informe de comités consultivos o mecanismos de consulta pública. La segunda responde a mecanismos no regulados donde el plan tiene modificaciones al margen de los mecanismos de participación ciudadana, pero que dan origen a un mecanismo de diálogo institucional con opacidad. Lo relevante es que, como se explicará más adelante, estas etapas informales tienen mayor incidencia en el resultado normativo del plan que las formales, lo que revela el problema de diseño regulatorio que tratamos de explicar en este trabajo.

\section{Las estaciones formales}

El presupuesto central sobre el cual descansa el proceso de elaboración de un plan es la declaración de una zona saturada o latente que requiere que una norma de calidad ambiental se encuentre sobrepasada o cercana a su superación. Para eso el Estado requiere de mediciones certificadas. ${ }^{12}$

Solo después de esto se puede iniciar la primera estación participativa de un plan, la elaboración del anteproyecto. ${ }^{13}$ Esta etapa, a su vez, tiene diversos momentos: a) el Ministerio de Medio Ambiente debe determinar cuáles son los antecedentes técnicos, científicos, económicos y/o sociales requeridos; b) se conforman los comités y subcomités operativos para la elaboración del plan, en donde participan representantes de ministerios, servicios públicos y otros organismos públicos competentes; c) se puede constituir un comité operativo ampliado, en donde además de los anteriores es posible integrar a representantes de la sociedad civil definidos a discreción por parte de

12. El reglamento que regula las estaciones de monitoreo para la validez de las mediciones es el Decreto Supremo 61, de 2008, del Ministerio de Salud Pública. Las auditorías de la Contraloría General de la República sobre cumplimiento de medidas de los planes evidencian problemas sobre la gestión de la red de estaciones de monitoreo, su administración, propiedad y funcionamiento, lo que, sin embargo, escapa al objeto de este trabajo. Ejemplo de dichas auditorías son el Informe Final 990-2018, de 15 de julio de 2020, sobre implementación de medidas del Plan de Descontaminación Atmosférica de Tocopilla; el Informe Final 529-2018, del 12 de septiembre de 2019, sobre las medidas asociadas al Plan de Descontaminación Atmosférica de Andacollo; el Informe Final 1502-2018, del 3 de enero de 2019, sobre el cumplimiento del Plan de Descontaminación Atmosférica de Talca.

13. Artículo 5 del Decreto Supremo 39, de 2012, del Ministerio del Medio Ambiente. 
la autoridad; d) en esa etapa cualquier persona puede entregar antecedentes técnicos, científicos, sociales y económicos adicionales sobre la zona; y e) la elaboración, a cargo del Ministerio del Medio Ambiente, de un análisis del impacto económico y social del anteproyecto de plan.

La segunda estación formal es la etapa de consulta pública. Esta tiene por propósito informar a la comunidad que se cuenta con un anteproyecto de plan, indicando el alcance geográfico del mismo, las medidas que se están proponiendo, un resumen de los fundamentos y el plazo para formular observaciones. Este último es nominalmente de sesenta días y las personas naturales o jurídicas pueden formular observaciones, las que deben estar - es lo que exige la regulación- acompañadas de los antecedentes que las sustenten, especialmente de naturaleza técnica, científica, social, económica y jurídica. ${ }^{14}$ Adicionalmente, el Ministerio del Medio Ambiente debe solicitar la opinión al Consejo Consultivo Nacional y Regional respectivo, quienes no se encuentran obligados a emitir un pronunciamiento.

Las regulaciones no establecen reglas sobre las metodologías o formas en que se deben llevar a cabo estos procesos participativos; estas quedan entregadas a la simple práctica administrativa, y que puede incluir audiencias públicas explicativas a nivel territorial, incluso en etapas previas a la elaboración de un anteproyecto ${ }^{15}$, pero en este caso de un modo completamente desformalizado, y sin que sea posible determinar su grado de influencia.

Terminada la etapa de consulta pública, la autoridad debe elaborar el proyecto final del plan, considerando los antecedentes del expediente y las observaciones de la etapa de consulta pública. ${ }^{16}$ Las normas ambientales nada dicen sobre el deber de respuesta a quienes hubiesen realizado observaciones en esta etapa, así como tampoco la obligación del Estado de explicar cuáles de las medidas adoptadas o corregidas en la versión final del proyecto del plan corresponden a observaciones o son consecuencia del proceso de consulta pública. ${ }^{17}$

Hasta acá llegan las estaciones formales. Luego de esto comienza una etapa en donde el plan continúa su procedimiento administrativo, pero el plan aún puede su-

14. Artículo 12 del Decreto Supremo 39, de 2012, del Ministerio del Medio Ambiente.

15. Etapas de participación ciudadana temprana - anteriores al anteproyecto- se han producido, por ejemplo, en la elaboración del Plan de Descontaminación Atmosférica por MPıo para las comunas de Talca y Maule, y de los Planes de Descontaminación Atmosférica para la comuna de Valdivia y de Coyhaique. Hasta ahora estas han sido promovidas como simples prácticas administrativas.

16. Artículo 13 del Decreto Supremo 39, de 2012, del Ministerio del Medio Ambiente.

17. Esto es aún más evidente si se considera que la jurisprudencia administrativa ha señalado que es posible que existan cambios a las medidas señaladas en el anteproyecto de plan y el Decreto Supremo que finalmente se publica, entendiendo que eso es fruto del procedimiento administrativo intermedio en donde pueden considerarse las observaciones de la comunidad. Véase el Dictamen 17.371 de la Contraloría General de la República, del 10 de mayo de 2001. 
frir modificaciones, muchas veces sustantivas, sin que exista obligación de consulta y menos una obligación de condicionar sus ajustes a lo señalado en las observaciones del procedimiento. Estas son las estaciones informales.

\section{Las estaciones informales}

La primera estacional informal es el Consejo de Ministros para la Sustentabilidad, institución creada por la reforma legal de 2009 que reestructuró la institucionalidad ambiental (Ley 20.417) ${ }^{18} \mathrm{y}$ que tiene la facultad de aprobar o rechazar la propuesta de plan elaborada. Si se aprueba, remite los antecedentes al Presidente de la República para su consideración final. Los antecedentes disponibles de las sesiones de dicho Consejo demuestran que en su interior no existen genuinas deliberaciones en torno a la información, los antecedentes recopilados y las observaciones formuladas en la elaboración del plan. ${ }^{19}$ Pero, además, en esta última etapa el Presidente puede realizar ajustes finales no considerados en las etapas procedimentales previas y sobre los cuales no existe escrutinio público alguno.

Como el plan de prevención y/o descontaminación se traduce en un decreto supremo del Presidente de la República y, como tal, está sometido obligatoriamente al trámite de toma de razón de la Contraloría General de República — que tiene por misión revisar la constitucionalidad y legalidad del plan,$-{ }^{20}$ en los hechos comienza la segunda estación informal. Esta no tiene regulación procedimental, por lo cual existe una práctica administrativa muy antigua, aplicable a todos los actos reglamentarios, de observaciones al texto remitido al trámite de toma de razón, que obliga a la autoridad a realizar retiros sucesivos del texto, ajustando su contenido y reingresándolo (De la Cruz Millar, 2019: 16). Esta práctica tiene cierta opacidad porque los ajustes requeridos no vuelven al Consejo de Ministros para la Sustentabilidad ni tampoco al Presidente de la República, sino que son simplemente una negociación entre la entidad contralora y el Ministerio de Medio Ambiente.

18. Mediante esta ley se creó el Ministerio del Medio Ambiente, el Servicio de Evaluación Ambiental y la Superintendencia del Medio Ambiente.

19. Como acreditó Horcos (2020), a diciembre de 2019, el Consejo de Ministros para la Sustentabilidad ha revisado planes en 29 sesiones, y alcanzó 17 acuerdos que versan o dicen relación con planes de prevención y/o descontaminación. El debate que se ha producido en esta instancia, en términos generales, no ha sido sustantivo. La mayoría de las intervenciones de los ministros y subsecretarios sectoriales tuvieron por finalidad solicitar prórrogas para el cumplimiento de algunas de las medidas o aclaraciones de los costos asociados a estos instrumentos.

20. Por ejemplo, en el caso del Plan de Descontaminación de Puchuncaví-Quintero (Decreto Supremo núm. 1, de 2017, del Ministerio del Medio Ambiente), la Contraloría objetó su contenido porque no cumplía con la finalidad que la normas establecen, toda vez que las medidas dispuestas por la autoridad, en opinión de Contraloría, no permitían una reducción efectiva de los contaminantes (véase Dictamen 44.528 de la Contraloría General de la República, del 16 de diciembre de 2017). 
Del análisis de siete planes recientemente, ${ }^{21}$ de un total de 561 artículos, 144 fueron observados por la Contraloría, la que realizó 318 reparos formales y sustantivos (Abalos Riquelme, 2018) provocando, en esta etapa, la incorporación de una gran cantidad de modificaciones a los textos de los planes, sin que existan antecedentes procedimentales que permitan realizar un escrutinio, como tampoco saber si tienen sustento en alguno de los trámites de la gestión del proyecto de cada plan.

El problema que se visualiza del contraste entre las estaciones formales e informales es evidente: son muchos más significativa e incidentes las modificaciones que se producen en los segundos espacios. De esta manera, se vulnera el principio participativo sobre el cual se construye la legislación ambiental.

\section{¿Quiénes participan?}

Para determinar los alcances de los sujetos que intervienen es conveniente caracterizarlos. Esto es útil porque nos permite observar el lugar al cual queda relegada la participación, atendida la incidencia de los sujetos que intervienen, sean estos públicos, los expertos o la comunidad en general.

En primer lugar, se encuentran los organismos públicos que tienen competencia o incidencia en las medidas asociadas al plan y que habitualmente se manifiestan en los denominados comités operativos. Bajo el modelo institucional de la Comisión Regional del Medio Ambiente (Corema), la gestión ambiental de los planes de prevención y/o descontaminación se confundía con el rol del Intendente como presidente de esta instancia. Con la reforma a la institucionalidad ambiental de $2009 \mathrm{y}$ la desaparición de esas comisiones regionales, se crearon las Secretarías Regionales Ministeriales del Medio Ambiente, lo que ha traído aparejado que la responsabilidad regional se vuelva ambigua. La indefinición de qué organismos participan da cuenta de la atomización institucional (agenzificación) de competencias ambientales, lo que implica costos significativos de coordinación. ${ }^{22}$

Por otra parte, está la participación de los expertos, especialmente del ámbito científico. Como ha demostrado la literatura, el rol de estos ha sido determinante en la lucha contra la contaminación atmosférica en nuestro país (Ibarra, O’Ryan y Silva, 2018), pero el diseño institucional no considera su participación activa u orgánica, más allá de la integración a los comités ampliados —una decisión completamente discrecional de la autoridad-, o bien, en la etapa de observaciones ciudadanas como

21. Corresponde a los planes de Valle Central de la Región del Libertador Bernardo O’Higgins (2013), Temuco Padre Las Casas (2015), Coyhaique (2015), Osorno (2015), Chillán y Chillán Viejo (2015), Talca y Maule (2015), y Valdivia (2016).

22. Véase Luis Cordero Vega, «El test de idoneidad para adoptar decisiones administrativas integradas», El Mercurio Legal, disponible en https://bit.ly/3hiPsJZ. 
cualquier interesado. Es más, el análisis del impacto económico y social no contempla ninguna participación formal de estos.

Finalmente, está la ciudadanía, que por sí misma es un conjunto variado de personas con muy distintos intereses, que tiene diversas representaciones según las regiones en donde se discuta un plan de prevención y/o descontaminación, lo que se explica no solo por la naturaleza de los planes, sino que también por el tipo de medidas. Mientras los planes del norte se vinculan a megafuentes, los del sur están fuertemente asociados a contaminación de fuentes domésticas y de industrias, tal como se explicitará más adelante.

\section{La evaluación de la participación}

De conformidad a la evidencia disponible, ${ }^{23}$ es posible considerar que quienes parti-

23. La metodología utilizada para analizar los procesos de consulta pública de los planes de prevención y/o descontaminación fue elaborada por Figueroa Meza (2019). Posteriormente, fue replicada por Quiero Benavente (2019), que agrupó los planes en las macrozonas norte, centro y sur. La metodología consiste en estudiar cada una de las observaciones (se eliminan las duplicadas y se desagrupan si no existe un tema central único) en base a cuatro ejes de análisis cualitativo que permite extraer conclusiones del proceso. Los ejes son los siguientes: i) temática, ii) contenido, iii) co-construcción, y iv) institucionalidad. La categoría temática busca responder a las preguntas: ¿Cuáles son los principales temas que preocupan a los participantes?, ¿hacia dónde orienta su interés? Se propone al identificar que los participantes, al manifestar su observación, se refieren a un tema central, con independencia de que en una observación puedan abordar una serie de temas (por ejemplo, leña o combustibles, sistemas de calefacción, vivienda, educación ambiental, salud, control de emisiones, fiscalización, entre otros). Se determinan en base a los contenidos propios del anteproyecto. El eje contenido pretende reflejar la subjetividad del participante, que se deja ver a través de su observación. Es así como se propone una serie de verbos que representen, en lo posible, la intención del participante al manifestar su observación: solicitar (el participante propone o solicita una medida para el plan, con o sin fundamentación, o solicita una acción concreta por parte de la autoridad en relación con el plan o su implementación), mejorar (el observante solicita la corrección de información o complementa una medida, lo que puede ser a través de una pregunta que sugiera un cambio o mejora, aunque no es así en todos los casos), complementar (el participante solicita mayor claridad o desarrollo de una medida o información al respecto, sobre todo en cuanto a la implementación de la medida), cuestionar (pone en duda la propuesta o incluso la rechaza, aunque no en forma explícita; cuestiona la eficiencia de la medida), rechazar (se manifiesta un desacuerdo total), reivindicar (se expresa una postura histórica o cultural), agradecer (se agradece la elaboración del plan y/o sus medidas; se destaca lo positivo); denunciar (a través de la observación el participante realiza una denuncia concreta hacia un sujeto o fuente de contaminación). Si bien en general la intención queda satisfecha con un solo verbo, en la mayoría de los casos se puede evidenciar un segundo verbo que refleja una intención subyacente o secundaria del participante. Para distinguir entre ambos se ha subdividido la categoría entre verbo primario y verbo secundario. La categoría co-construcción es la que analiza propiamente la incidencia, toda vez que, tomando como base el contenido manifestado por el participante, se determina si el sujeto tenía como objetivo incidir en la determinación del texto del plan, esto es, si asume un rol activo como co-diseñador del instrumento. Este eje responde a la pregunta: 
cipan en la etapa de consulta pública son personas naturales y jurídicas, mujeres y hombres, cuyo porcentaje variará dependiendo de la zona en la que se esté elaborando el plan. En efecto, es posible distinguir entre la zona norte, central y sur para caracterizar la participación. En el caso de las personas jurídicas, estas son regularmente incumbentes u organizaciones sociales en la defensa de un interés ambiental. En el caso de las personas naturales son de muy diversa entidad, y podríamos encontrar desde aquellas que tienen una preocupación por su afectación en el modo de vida hasta personas con una vocación de protección ambiental.

Respecto de las observaciones, esta es quizá una de las áreas en donde se evidencia con mayor nitidez la distancia entre la pretensión normativa y su ejecución. De acuerdo con los datos analizados, las observaciones se caracterizan por requerir más información al regulador sobre medidas, desconfiar de su utilidad o informar sobre situaciones históricas. La mayoría de ellas no tienen que ver con el contenido de las medidas de los planes, sino que sostienen opiniones sobre cuestiones vinculadas a la calidad de vida de las zonas afectadas (Quiero Benavente, 2019). En ese contexto, la distribución normativa - que exige acompañar antecedentes y que, como señalábamos, opera como una restricción de acceso- no se estaría aplicando en la práctica, pero la norma subsiste, asume un nivel de conocimiento de los ciudadanos y olvida las asimetrías inevitables que derivan de la complejidad de los problemas ambientales. Esto tiene implicancias para futuras reformas legales en esta materia.

En términos generales, el número de participantes frente a la población total de las comunas reguladas, en cada uno de los planes, resulta particularmente baja: en la mayoría de los casos es menos del $1 \%$ de la población, como se aprecia de la tabla 1. Lo anterior podría encontrar su explicación en problemas de difusión de la informa-

\footnotetext{
¿El participante utiliza la consulta con la pretensión de incidir en la elaboración del plan, modificando o co-diseñando una medida? Por último, la categoría institucionalidad persigue estudiar el diálogo que se produce entre participantes y autoridades, identificando si los primeros reconocen la labor de los organismos públicos involucrados y los interpelan, o si bien, no hacen mención a estos y no existe reconocimiento explícito de la autoridad. Para ello, se plantean las preguntas: ¿El participante menciona explícitamente a la autoridad administrativa?, ¿cómo se refiere a ella? Cabe aclarar que se entiende que el participante está dirigiéndose a la autoridad administrativa, pero solo se ha considerado positiva la respuesta a la primera pregunta cuando identifica con claridad el órgano, servicio, ministerio, etcétera. En relación con la segunda pregunta, que será contestada solo en aquellos casos en que la primera haya sido respondida afirmativamente, se han establecido una serie de verbos que representan el modo en que el participante se refiere a la Administración, lo que solicita de ella, a saber: regular (requiere de la autoridad que esta elabore, implemente o profundice una medida), fiscalizar (solicita verificar el cumplimiento de una medida o su implementación por los regulados), subsidiar (se requiere que el Estado asuma el costo de implementación de una medida), financiar (se plantea la inquietud sobre el origen de los recursos financieros para costear una medida), coordinar (se solicitó la coordinación de los órganos administrativos, en relación a la implementación de una medida), evaluar (plantea la necesidad explícita de evaluación).
} 
Tabla 1. Cantidad y tipos de personas que participan

\begin{tabular}{|c|c|c|c|c|c|c|c|}
\hline & Plan & $\begin{array}{l}\text { Personas } \\
\text { naturales }\end{array}$ & $\begin{array}{l}\text { Personas } \\
\text { jurídicas }\end{array}$ & $\begin{array}{l}\text { Total de } \\
\text { personas }\end{array}$ & $\begin{array}{l}\text { Total de } \\
\text { observaciones }\end{array}$ & $\begin{array}{l}\text { Población } \\
\text { (habitantes) }\end{array}$ & $\begin{array}{l}\text { Superficie } \\
\text { en } \mathrm{km}^{2}\end{array}$ \\
\hline \multirow{3}{*}{$\begin{array}{l}\text { Macrozona } \\
\text { Norte }\end{array}$} & Tocopilla & 3 & 6 & 9 & 80 & 23.986 & 4.038 \\
\hline & Andacollo & 7 & 2 & 9 & 104 & 8.300 & 310 \\
\hline & Huasco & 6 & 4 & 10 & 83 & 10.429 & $\mathrm{~N} / \mathrm{A}$ \\
\hline \multirow{2}{*}{$\begin{array}{l}\text { Macrozona } \\
\text { Central }\end{array}$} & Santiago & 526 & 52 & 578 & 1227 & 7.140 .674 & $15.554,5$ \\
\hline & $\begin{array}{l}\text { Concón, Quintero y } \\
\text { Puchuncaví }\end{array}$ & 223 & 28 & 251 & 793 & 92.621 & $\mathrm{~N} / \mathrm{A}$ \\
\hline \multirow[t]{10}{*}{$\begin{array}{l}\text { Macrozona } \\
\text { Sur }\end{array}$} & $\begin{array}{l}\text { Valle Central de la } \\
\text { Región del Libertador } \\
\text { General Bernardo } \\
\text { O'Higgins }\end{array}$ & 14 & 22 & 36 & 284 & 688.364 & 3.443 \\
\hline & Talca-Maule & 59 & 3 & 62 & 90 & 280.298 & 469,7 \\
\hline & $\begin{array}{l}\text { Valle Central de la } \\
\text { Provincia de Curicó }\end{array}$ & 60 & 16 & 76 & 165 & 222.494 & 2.117 \\
\hline & Concepción & 196 & 23 & 219 & 483 & 971.285 & 2.102 \\
\hline & Chillán y Chillán Viejo & 24 & 2 & 26 & 73 & 184.037 & 803 \\
\hline & Los Ángeles & 131 & 10 & 141 & 194 & 215.389 & 1.748 \\
\hline & Temuco & 252 & 23 & 275 & 425 & 339.664 & 929,5 \\
\hline & Valdivia & 20 & 4 & 24 & 120 & 154.097 & $1.015,60$ \\
\hline & Osorno & 62 & 9 & 71 & 140 & 145.475 & 6.805 \\
\hline & Coyhaique I & 65 & 6 & 71 & 422 & 50.041 & 7.775 \\
\hline
\end{tabular}

Fuente: Elaboración propia a partir de expedientes públicos de tramitación.

ción y de educación ambiental, y ausencia de herramientas adecuadas, generados por una brecha de conocimiento, que podría limitar el acceso de determinados grupos de la población. En la actualidad no existe ningún estudio que explique las razones de la ausencia de participación en los planes de prevención y/o descontaminación en particular, pero la jurisprudencia es consistente en señalar que le corresponde a la Administración del Estado fomentar, promover y fortalecer esas instancias. ${ }^{24}$

Es posible diferenciar el tipo de participación dependiendo de las macrozonas. Respecto de la macrozona norte, es posible que la emisión de material particulado tenga relación con las actividades industriales y mineras. Esto se traduce en que el

24. «Para la jurisprudencia de la Corte Suprema, la participación ciudadana es un principio estructural, junto con el de prevención y precaución, en la construcción de la narrativa tutelar que cumple la legislación ambiental» (Luis Cordera Vega, «Modelando la participación ciudadana en el SEIA», 26 de febrero de 2021, disponible en https://bit.ly/35Yt2IK). 
inventario de emisiones, contenido en los antecedentes de cada plan, determina específicamente quiénes son los responsables y cuántas emisiones relacionadas a sus procesos productivos generan. Esto incide directamente en la cantidad de participantes que plantean observaciones a las medidas adoptadas por la autoridad, en el instrumento de gestión: es determinante la participación de personas jurídicas, en particular de carácter privado, pues son quienes se ven principalmente afectadas con el instrumento. Así, ninguno de estos planes supera el total de diez observantes. En cuanto a los contenidos propiamente tal, de las observaciones realizadas a los planes, particularmente relevante son las tasas de cuestionamientos a sus medidas, que alcanzan el 30\%. El rechazo varía entre el 1\% y 19\% de las observaciones, lo que revela la gran cantidad de observaciones que tienen un componente negativo en torno a las medidas que son planteadas.

En el caso de la macrozona central, la participación de las personas naturales es mayoritaria, distribuyéndose homogéneamente entre mujeres y hombres. En términos de su contenido, el verbo rector predominante de las observaciones es cuestionar (aprox. 22\%), seguido por el rechazo a las medidas (20\%). Cabe resaltar la dimensión de co-construcción, pues del total de observaciones de los planes analizados, es la macrozona en la que más relevancia tiene: alcanza un promedio de $49 \%$. Más sorprendente es que en la participación total de personas jurídicas, en lo que concierne a esta dimensión, las cifras alcanzan el $75 \%$, y es indistinto si son públicas o privadas.

Por último, en el caso de la macrozona sur, la participación tiene evidente relación con las características que determinan los problemas de contaminación ambiental, pues las medidas, en general, se focalizan en los esfuerzos regulatorios de la leña. Lo anterior conlleva a que el principal regulado por estos instrumentos de gestión ambiental sean personas naturales, lo que aumenta el índice de participación, pues la mayoría de las medidas tienen relación con el uso de la leña como combustible y los recambios de los sistemas de calefacción. Producto de ello, las observaciones no se focalizan tanto en cuestionar las medidas, sino más bien en complementarlas (para mejorar la regulación) o en solicitar más información.

En términos de efectividad de la participación, es posible afirmar que no existe una incidencia real de la ciudadanía en el texto del plan (Quiero Benavente, 2019; Figueroa Meza, 2019; Acevedo Espinoza y Fuenzalida Peñaloza, 2018), lo que podría explicarse por los problemas antes mencionados, pero también por la postura de los participantes, que no asumen dentro del proceso un rol co-constructor. ${ }^{25}$ En efecto, son escasas las observaciones que tienen una pretensión de modificación de la redacción del articulado del plan, y muchas de las observaciones evidencian una desconfianza respecto de la institucionalidad y el mecanismo de elaboración del instrumento.

25. Véanse las referencias al rol co-constructor de la ciudadanía en Montedonico y otros (2018: 5). 


\section{Conclusiones}

Es evidente el desajuste entre lo pretendido al momento de establecer las reglas jurídicas y lo que muestra el grado de participación ciudadana en materia de planes de prevención y/o descontaminación. Teniendo en consideración lo expuesto, es posible afirmar que no hay un genuino sistema de participación ciudadana ambiental en los planes. La figura de la consulta pública es insuficiente, la carga para los que proveen de observaciones es inadecuada y el Estado no gestiona las observaciones levantadas a través del proceso. Los antecedentes recopilados dan cuenta que de los cincos aspectos para evaluar un proceso participativo - liderazgo en el proceso participativo, enfoque holístico de la política pública sometida a participación, amplia visibilidad al proceso participativo, capacidad de impacto de los ciudadanos en las políticas públicas a través del proceso de participación, y cambio cultural en políticos, técnicos y ciudadanos-, los planes de prevención y/o descontaminación no calificarían satisfactoriamente en ninguno.

En efecto, el proceso de consulta pública no impone la obligación al Estado, como sí sucede en el Sistema de Evaluación de Impacto Ambiental, de ponderar las observaciones obligatoriamente, ${ }^{26}$ lo que - como se sabe - tiene importantes efectos desde la perspectiva de acceso a la justicia ambiental; no en cuanto a la legitimación activa, sino a la pretensión jurídica. La impugnación a través de la reclamación contenida en la Ley 20.600 tendrá como estándar de revisión lo señalado en la Ley 19.300, lo que implica concentrarse en los aspectos formales, más no en idoneidad de las medidas o el contenido de las observaciones efectuadas en la consulta pública, lo que reduce ciertamente las posibilidades de éxito y la vinculación del observante con el procedimiento jurisdiccional. La consecuencia es bastante inevitable: la pretensión de información y legitimidad social que se busca a través de mecanismos de consulta simplemente formales, están lejos de cumplir su objetivo.

Pero, además, existe una importante cantidad de información obtenida a través de los procesos de consulta que, si bien no se vincula a los aspectos propios de los planes, sí provee de antecedentes útiles para la gestión ambiental general del territorio en donde estos procesos se desarrollan y para el cumplimiento de sus medidas.

Una regla que obligue al Estado a sistematizar esa información y dejarla a disposición permanente de la ciudadanía puede ser útil para orientar la gestión de los planes, así como otras políticas aplicables en el territorio en términos integrados, porque es

26. Esto es bien determinante en la jurisprudencia judicial. La Corte Suprema ha establecido un estándar para la ponderación de observaciones que permita una genuina participación al señalar que «la debida consideración de las observaciones no consiste en la existencia de una posibilidad de análisis, sino en el efectivo estudio de la consulta» (Corte Suprema, Carrera con Servicio de Evaluación Ambiental, 2 de julio de 2019, rol 28.207-2018, considerando undécimo). 
la única forma de delimitar la capacidad de impacto de los procesos participativos y propiciar el cumplimiento de las medidas del instrumento.

Por último, otro de los elementos que se aprecia en relación con el diseño normativo es la ausencia de la ciencia en términos institucionales al momento de generar la evidencia y participar en los asuntos vinculados a los planes de prevención y/o descontaminación. Aunque como han señalado otros estudios, la participación de científicos ha sido importante en la obtención de resultados positivos en materia de descontaminación atmosférica (Ibarra, O’Ryan y Silva 2018), esta ha carecido de un componente orgánico.

Por ejemplo, la participación institucional de la ciencia no se encuentra establecida en la Ley 19.300 sobre Bases Generales del Medio Ambiente, salvo en la posibilidad de integrar el Consejo Consultivo Nacional y los Consejos Regionales de Medio Ambiente; pero tampoco la ciencia institucionalmente tiene responsabilidad en la elaboración de los estudios para el anteproyecto de los planes de prevención y/o descontaminación, ni menos en la evaluación de los impactos económicos y sociales, salvo mediante la contratación de consultorías para tales fines. En materia de implementación y seguimiento, esa posibilidad es sencillamente remota de acuerdo con las reglas actuales.

El establecimiento de un sistema orgánico de participación de la ciencia en la política pública en contaminación atmosférica resulta central para el proceso que debemos continuar. Si bien los datos son positivos en cuanto la disminución de la contaminación en los últimos veinte años (O’Ryan e Ibarra, 2017), el desafío que enfrentamos es estructuralmente difícil, de modo que un modelo más complejo de participación debe reconocer a la ciencia en términos activos y no circunstanciales en el caso del diseño y gestión de los planes de prevención y/o descontaminación.

Así las cosas, resulta inevitable modificar tanto la Ley 19.300 sobre Bases Generales del Medio Ambiente y las normas reglamentarias de ejecución a fin de garantizar un mejor acceso de la ciudadanía a los procedimientos administrativos ambientales asociados a los planes de prevención y/o descontaminación, y aeegurar la presencia de la ciencia en el ciclo regulatorio, creando mecanismos que permitan la co-construcción de estos instrumentos. Todo esto incidirá en su legitimidad, implementación, cumplimiento y, en consecuencia, en su eficacia.

\section{Referencias}

Acevedo Espinoza, Javiera y Fuenzalida Peñaloza, Juan Pablo (2018). La participación ciudadana en los planes de prevención y descontaminación ambiental. Memoria para optar al grado de Licenciado en Ciencia Jurídicas y Sociales, Facultad de Derecho, Universidad de Chile. Disponible en http://repositorio.uchile.cl/ handle/2250/153076. 
Abalos Riquelme, Javiera (2018). El control de legalidad ejercido por la Contraloría General de la República en Planes de Descontaminación. Memoria para optar al grado de Licenciado en Ciencia Jurídicas y Sociales, Facultad de Derecho, Universidad de Chile.

Astorga Jorquera, Eduardo (2014). Derecho Ambiental chileno. $4 .^{a}$ edición. Santiago de Chile: Thomson Reuters.

Bermúdez Soto, Jorge (2015). Fundamentos de Derecho Ambiental. 2. ${ }^{a}$ edición. Valparaíso: Ediciones Universitarias de Valparaíso.

CORPORACIÓN TIEMPO 2000 (1999). Los planes de descontaminación y normas de calidad y emisión. Balance de una década, serie ambiental. Santiago: Corporación Tiempo 2000.

De la Cruz Millar, Alicia (2019). Contraloría General de la República: ¿Jurisdicción contenciosa administrativa? Santiago: Der Ediciones.

DuRÁN, Valentina, Lorena Schmitt, Claudia Sepúlveda y Rodrigo Wagner (2017). «Del conflicto al diálogo: Cómo avanzar hacia un sistema de decisiones ambientales participativas en Chile». Espacio Público. Disponible en https://bit.ly/3hhkMce.

Figueroa Meza, Susana (2019). Análisis de la participación ciudadana en el Plan de Descontaminación Atmosférica por Mpio de la Ciudad de Coyhaique y su zona circundante. Tesis de Maestría, Escuela de Postgrado, Facultad de Derecho, Universidad de Chile.

Guzmán Rosen, Rodrigo (2012). Derecho Ambiental chileno. Santiago: Planeta Sostenible.

Hervé Espejo, Dominique (2010). «Noción y elementos de la justicia ambiental: Directrices para su aplicación en la planificación territorial y en la evaluación ambiental estratégica». Revista de Derecho (Universidad Austral de Chile), 23 (1), 9-36. DOI: 10.4067/S0718-09502010000100001.

Horcos, Benjamín (2020). El rol del Consejo de Ministros para la Sustentabilidad en la elaboración de los Planes de Prevención y Descontaminación Atmosférica entre 2013 y 2018. Memoria para optar al grado de Licenciado en Ciencias Jurídicas, Facultad de Derecho, Universidad de Chile.

IBARRA, C., R. O'Ryan y B. Silva, B. (2018). Applying knowledge governance to understand the role of science in environmental regulation: The case of arsenic in Chile. Environmental Science \& Policy, 86: 115-124, DOI: 10.1016/j.envsci.2018.05.002.

INNES, E. y D. E. Booher (2004). «Reframing public participation: Strategies for the $21^{\text {sT }}$ Century». Planning Theory \& Practice, 5 (4): 419-436.

LeE, Maria y Carolyn Abbot (2003). «The usual suspects? Public participation ander the Aarhus Convention». Modern Law Review, 66 (1): 80-108. DOI: 10.1111/1468-2230.6601004.

Linares, Sebastián (2017). Democracia participativa epistémica. Madrid: Marcial Pons. 
Montedonico, M., F. Herrera-Neira, A Marconi y Urquiza. (2018). «Co-construcción en proyectos de generación distribuida con energía solar: participación de la comunidad en el proyecto Ayllu Solar». Revista Estudios Avanzados, 29: 4-22. Disponible en https://bit.ly/3jtgvs2.

OCDE y Cepal (2005). Evaluación del desempeño ambiental. Santiago: Naciones Unidas y Cepal.

O'Ryan, Raúl y Cecilia Ibarra (2017). «Environmental policy in Latin America». En A. Farazmand (editor), Global Encyclopedia of Public Administration, Public Poli$c y$, and Governance. Cham: Springer International Publishing.

PArÉs, M., A. Ballester, J. Espluga y Q. Brugué (2014). «Gobernanza deliberativa en la gestión de cuencas hidrográfica: Analizando las consecuencias de la directiva marco del agua en Cataluña». Investigaciones Geográfica, 61. DOI: 10.14198/ INGEO2014.61.08.

Pintaudi, Silvana (2005). «Participación ciudadana en la gestión pública». Scripta Nova, 9 (194). Disponible en http://www.ub.edu/geocrit/sn/sn-194-102.htm.

Quiero Benavente, Débora (2019). Análisis crítico de los procesos de participación ciudadana en los Planes de Prevención y Descontaminación Atmosférica. Los casos de Talca y Chillán. Memoria para optar al grado de Licenciado en Ciencias Jurídicas, Facultad de Derecho, Universidad de Chile.

Rowe, Gene y Lynn Frewer (2000). «Participation methods: A framework for evaluation». Science, Technology, \& Human Value, 25 (1):3-29. DOI: 10.1177/016224390002500101

Soto Barrientos, Francisco y Costa Cordella, Ezio (2019). «Tensiones y desafíos de la participación ciudadana en materia ambiental». Revista Derecho del Estado, 44. Disponible en 10.18601/01229893.n44.09.

WALKer, Gordon (2012). Enviromental justice. Londres: Routledge.

\section{Reconocimiento}

Este artículo ha sido escrito en el marco de los proyectos Fondecyt Regular 1170767 y ANID/Fondap/15110009 del Centro de Ciencia del Clima y la Resiliencia (CR2).

\section{Sobre los autores}

Luis Cordero Vega es doctor en Derecho por la Universidad de Lleida (España). Es profesor de Derecho Administrativo en la Facultad de Derecho de la Universidad de Chile e investigador del Centro de Ciencia del Clima y la Resiliencia (CR2). Su correo electrónico es lcordero@derecho.uchile.cl. (D) https://orcid. org/0000-0002-2967-5074. 
Ximena Insunza Corvalán es magíster de la McGill University (Canadá) y magíster en Políticas Públicas de la Universidad de Chile. Es profesora del Departamento de Derecho Económico e investigadora del Centro de Derecho Ambiental de la Facultad de Derecho de la Universidad de Chile. Su correo electrónico es xinsunza@ derecho.uchile.cl. (DD https://orcid.org/oooo-0002-2943-1459. 
La Revista de Derecho Ambiental, del Centro de Derecho Ambiental de la Facultad de Derecho de la Universidad de Chile, es un espacio de exposición y análisis en el plano académico del derecho ambiental. Su contenido se presenta a través de doctrina, jurisprudencia y recensiones, y aborda diversas materias relacionadas con la gestión, institucionalidad y herramientas de protección ambiental y desarrollo sustentable. Se presentan artículos de diferentes autores y autoras en los que se analizan y abordan casos y temas jurídico-ambientales de creciente interés y actualidad.

\author{
DIRECTORA \\ Valentina Durán Medina \\ EDITORES \\ Jorge Ossandón Rosales \\ y Antonio Pulgar Martínez \\ SITIO WEB \\ revistaderechoambiental.uchile.cl \\ CORREO ELECTRÓNICO \\ revistada@derecho.uchile.cl \\ LICENCIA DE ESTE ARTÍ́CULO \\ Creative Commons Atribución Compartir Igual 4.o Internacional
}

La edición de textos, el diseño editorial

y la conversión a formatos electrónicos de este artículo

estuvieron a cargo de Tipográfica

(www.tipografica.io) 